\title{
Bullous Mastocytosis in a Filipino Infant: A Case Study
}

\author{
Blythe N. Ke, MD, MBA, Shahara Abalos-Babaran, MD, Jay-V James G. Barit, MD, \\ Mia Katrina R. Gervacio, MD and Mae N. Ramirez-Quizon, MD \\ Department of Dermatology, Philippine General Hospital, University of the Philippines Manila
}

\begin{abstract}
Introduction. Mastocytosis is a disease defined by the proliferation of mast cells in organs, most commonly the skin. It may affect any age group but is usually found in children in the first year of life. We present a case of diffuse cutaneous mastocytosis manifesting in the rare bullous form.

Case. A 4-month-old Filipino male presented with multiple bullae on the head, trunk, and extremities after applying chamomile oil. Biopsy of the skin demonstrated numerous mast cells, confirming the diagnosis of bullous mastocytosis. The patient was treated with oral antihistamines and corticosteroids, which was followed by a good response.
\end{abstract}

Conclusion. Diagnosis of diffuse cutaneous mastocytosis may be challenging due to its rarity. Proper management requires preventive measures, symptomatic treatment, as well as communication of prognosis with the stakeholders.

Key Words: bullous mastocytosis, cutaneous mastocytosis, diffuse cutaneous mastocytosis

\section{INTRODUCTION}

Paper presented in the $42^{\text {nd }}$ Philippine Dermatological Society Annual Convention and $10^{\text {th }}$ Asian Society for Pigment Cell Research Congress, on November 5-7, 2019, at the Shangri-La Hotel, Mandaluyong City, Metro Manila, Philippines.

Corresponding author: Blythe N. Ke, MD, MBA

Department of Dermatology

Philippine General Hospital

University of the Philippines Manila

Taft Avenue, Ermita, Manila 1000, Philippines

Email: bnke2@up.edu.ph
Mastocytosis is defined as an abnormal accumulation of mast cells in one or more organs. The human adult has an estimated 75 mast cells per $\mathrm{mm}^{2}$ of skin, with higher concentrations in the distal extremities. ${ }^{1,2}$ In cutaneous mastocytosis, these levels increase by 4 to 8 times the normal. ${ }^{2}$

Cutaneous mastocytosis (CM) usually appears in the first few years of life and generally disappears during puberty. ${ }^{2}$ The three types of CM are maculopapular/urticaria pigmentosa (UP), localized mastocytoma, and diffuse cutaneous mastocytosis (DCM). Among these, DCM is the most uncommon, with lesions appearing as tan-brown papules, nodules, and plaques; occasionally, thickened skin with a peau d'orange appearance is observed. ${ }^{3}$ DCM may also present as hemorrhagic blisters all over the body and this subtype, known as bullous mastocytosis (BM), is the rarest form.

In a systematic review of 1747 pediatric mastocytosis cases, $5 \%$ of the cases were DCM, and only $50 \%(n=44)$ of these cases were bullous. ${ }^{4}$ Another review of 173 cases of pediatric mastocytosis by Hannaford and colleagues reported only three cases of DCM, all of which were bullous. ${ }^{5}$ Up until 2018, there are only 63 cases of BM reported in the literature. ${ }^{6}$ Meanwhile, local data spanning 2011 to 2018 from the Philippine Dermatological Society Health Information System reported 57 cases of cutaneous mastocytosis, of which only seven were bullous. ${ }^{7}$ 


\section{CASE PRESENTATION}

A 4-month-old previously healthy male was brought into the emergency room for multiple tense bullae and erosions on the scalp, trunk, and upper extremities. This was noted an hour after application of aceite de manzanilla (chamomile oil) to his abdomen, apparently as a home remedy for his vomiting. There were previous applications of the same oil without any untoward reactions. The patient was born without fetomaternal complications and received age-appropriate immunization. Family history is positive for asthma (maternal); however, there were no notable skin conditions.

On physical examination, the patient was afebrile and presented with multiple tense bullae with areas of erosions on the scalp, trunk, and upper extremities (Figure 1A and 1B). Hands, feet, and mucosal surfaces were spared. Complete blood count showed anemia and leukocytosis, while
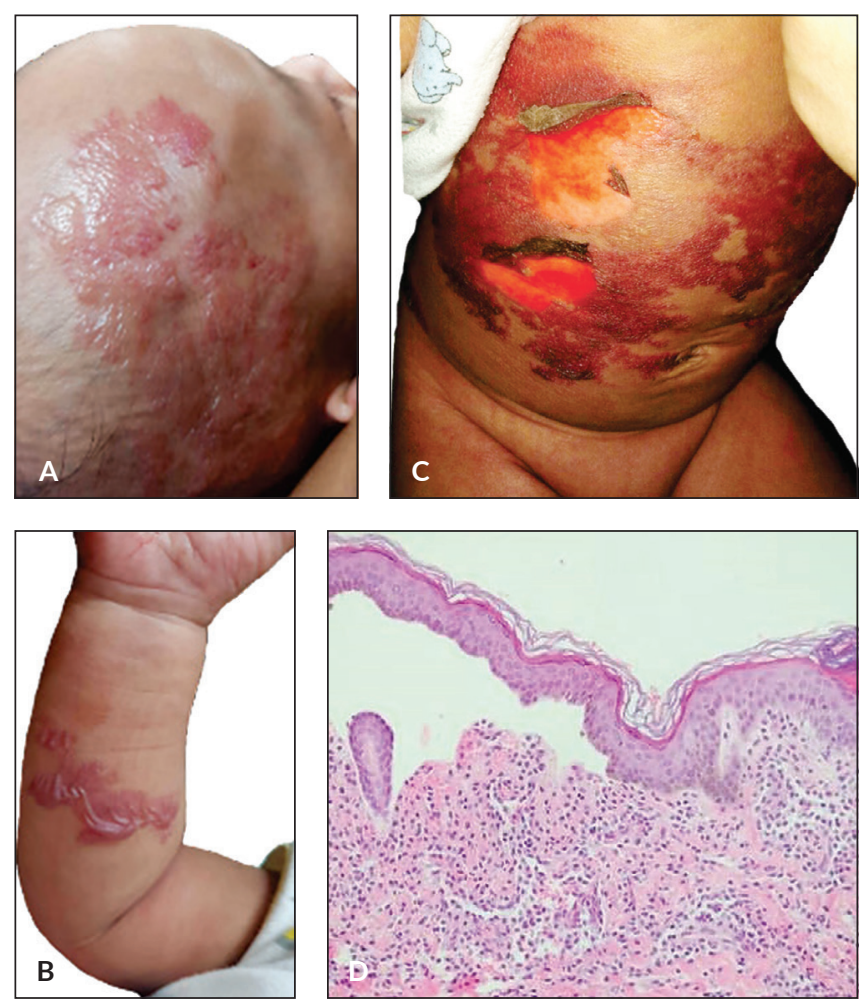

Figure 1. Patient on presentation at the emergency room. (A) Scalp and (B) upper extremity show multiple erythematous plaques (peau d'orange appearance) and bullae. (C) Abdomen with erythematous and hemorrhagic plaques, significant erosions, and few confluent hemorrhagic vesicles at the lower portion. (D) Biopsy of an abdominal vesicle showed basketweave orthokeratosis, mild spongiosis, subepidermal split with marked dermal edema, and lichenoid infiltrate lymphocytes, occasional eosinophils, and few mast cells (Hematoxylin and eosin, 10x). prothrombin time was prolonged. Blood culture was negative. Biopsy of an abdominal vesicle (Figure 1C) revealed mild spongiosis, subepidermal split, and marked dermal edema. There were lichenoid infiltrate of lymphocytes, occasional eosinophils, and a few mast cells (Figure 1D). At this point, allergic contact dermatitis from a possible prior sensitization, or irritant contact dermatitis, perhaps from a higher quantity of applied oil, was considered. Although the oil was reportedly applied to the abdomen, it was presumed to have come into contact with the patient's scalp and extremities while caregivers were handling him. Management was performed in a burn unit where intravenous hydrocortisone was given, and sterile dressings were applied. The patient's caregivers were advised to avoid the use of the suspected irritant.

During the hospital stay, lesions decreased significantly but recurred on the $8^{\text {th }}$ day, where multiple tense bullae were again seen on the head (Figure 2A), trunk, and extremities of the patient. Biopsy was repeated on a bulla on the thigh
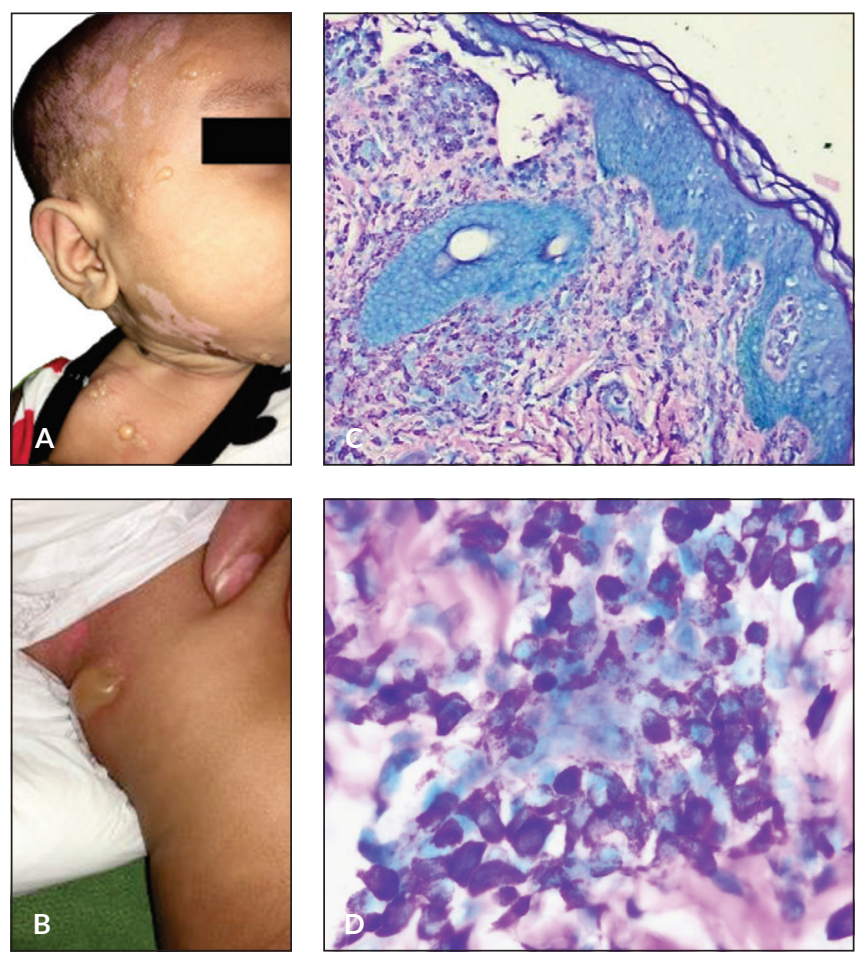

Figure 2. (A) Patient with recurrence of tense bullae at the face and neck. Hypopigmented patches from previously resolved bullae are seen. (B) Tense bulla with surrounding faint erythema is noted at the patient's medial thigh. (C) Biopsy done on the bulla at the medial thigh showed basketweave orthokeratosis, subepidermal split, dense to superficial perivascular, periadnexal, and interstitial infiltrates of numerous mast cells, lymphohistiocytes, eosinophils, and neutrophils (Giemsa stain, 40x). (D) A closer view revealed the numerous mast cells in the dermis with highlighted granules (Giemsa stain, 100x). 

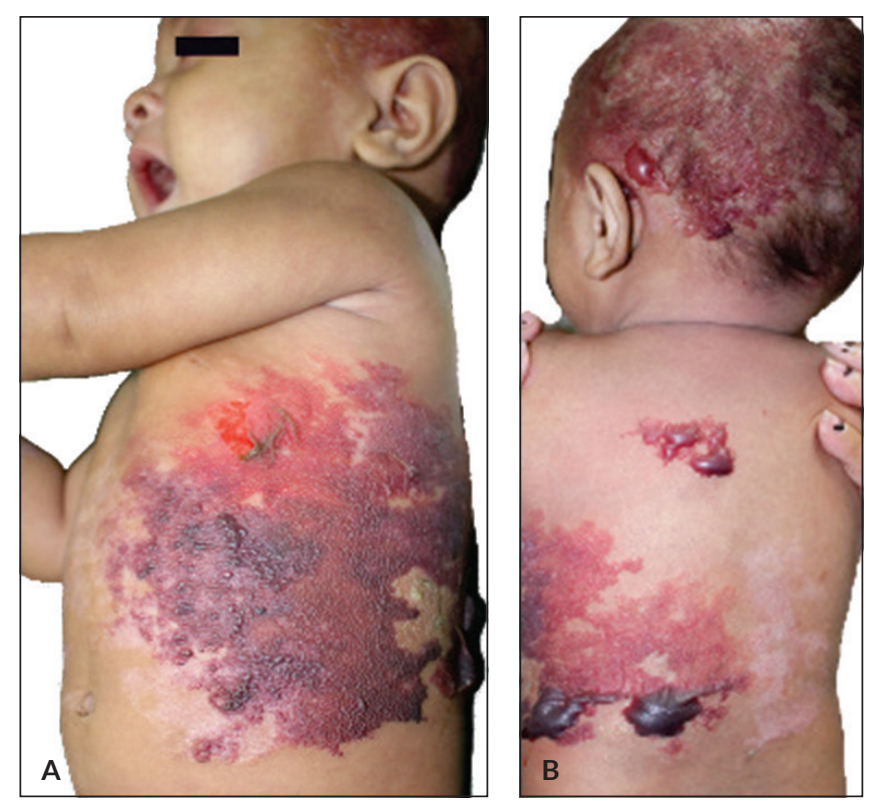

Figure 3. Views of the (A) lateral and (B) posterior trunk showed post-inflammatory hypopigmentation and hemorrhagic vesicles and bullae on patient's followup consultations.

(Figure 2B), which showed subepidermal split and dense superficial to deep perivascular, periadnexal, and interstitial mixed cell infiltrates. On Giemsa stain (Figure 2C and 2D), numerous mast cells were highlighted at the dermis. Direct immunofluorescence (DIF) was negative, making the diagnosis compatible with diffuse cutaneous mastocytosis of the bullous type. The patient was given cetirizine, antibacterial ointment, and saline compresses which led to the resolution of the lesions. Caregivers were then educated regarding the nature and prognosis of the disease and were instructed to avoid any forms of trauma to the patient's skin.

On follow-up consultations, occasional recurrence of hemorrhagic bullae on the head and body (Figure 3A and 3B) were managed with $\mathrm{H} 1$ antihistamines (cetirizine), topical corticosteroids (hydrocortisone), and bland emollients. Caregivers were regularly reminded regarding avoidance of triggers and the importance of regular follow-up consultations.

\section{DISCUSSION}

\section{Etiology and Pathogenesis}

In mastocytosis, a gain of function mutation in the c-kit receptor is implicated, which codes for a tyrosine kinase receptor for mast cell growth factor. ${ }^{6}$ The pathogenesis of CM is not fully understood as not all children present with this mutation. ${ }^{2}$ The symptoms of the disease arise due to the release of preformed mediators, newly synthesized mediators, and cytokines, potentially resulting in vasodilation, gastrointestinal symptoms, and bone pain. Darier's sign may be elicited by scratching or rubbing the skin. ${ }^{3}$

\section{Classification and Diagnosis}

The previous classification of mastocytosis created by WHO in 2001 separates the disease into cutaneous, indolent systemic, systemic, and associated clonal nonmast cell lineage disease (SM-AHNMD), aggressive systemic, and extracutaneous forms. ${ }^{8}$ However, a more straightforward classification system was released in 2016, giving rise to cutaneous, systemic, and mast cell sarcoma. Here, the cutaneous form is subdivided into maculopapular CM (urticaria pigmentosa), diffuse CM, and localized mastocytoma. ${ }^{9}$

Diagnosis of $\mathrm{CM}$ and its differentiation from SM and other bullous diseases consists of performing a clinical examination, laboratory tests, and histopathologic studies. Performing the Darier's sign, an urticarial reaction elicited by stroking or rubbing skin lesions is a helpful diagnostic feature to distinguish from other bullous diseases. Still, it should be done with caution as it may induce hypotension. ${ }^{2} \mathrm{CM}$ patients may present with pruritus, flushing, abdominal pain, and diarrhea due to the release of mediators by cutaneous mast cells; however, these do not necessarily signify systemic involvement. Suspicion of systemic involvement, especially in adults, warrants a thorough physical examination to assess for hepatosplenomegaly and lymphadenopathy and an extensive workup that may involve bone marrow biopsy, abdominal imaging, and genetic testing. ${ }^{9}$ As most pediatric mastocytosis is limited to the skin (90\%), the diagnosis of $\mathrm{CM}$ may be assumed without routinely performing bone marrow biopsy. ${ }^{10,11}$ In the patient's case, only a punch biopsy of the skin and few blood tests were performed. The relevant findings of leukocytosis and prolonged prothrombin time were attributed to the effect of mast cell mediators. If available, tryptase levels should be obtained as it correlates closely with the disease course and severity. ${ }^{12}$

Histopathologic findings for cutaneous mastocytosis include clustered proliferation of mast cells, usually in the papillary dermis. The neoplastic cells are metachromatic upon staining with Giemsa or toluidine blue. In addition, immunohistochemical staining with CD117 (c-Kit), chymase, tryptase, and Leder may be utilized to confirm mast cell identity and distribution. ${ }^{13,14}$

The differential diagnosis of BM includes an array of autoimmune, infectious, and inflammatory conditions, leading to misdiagnosis. ${ }^{10}$ Due to the rarity of BM locally, it is not uncommon to consider the diagnosis only after other diseases have been ruled out. Such was the case above where initial biopsy only revealed polymorphic cell infiltrates, and few mast cell counts, subsequently necessitating the investigation for allergic/irritant, infectious, and immune etiologies. A possible reason for this non-diagnostic finding is mast cell degranulation in the initial biopsy site, making histopathologic examination more difficult. Although a vesicular area was targeted for biopsy at the abdomen, most of the surrounding skin appears to have already been traumatized and eroded. 
Furthermore, direct injection of local anesthetic with epinephrine into the biopsy site should preferably be avoided as it may also cause degranulation. Some authors advise injecting the local anesthetic without epinephrine below or adjacent to (rather than directly into) the chosen biopsy site. $^{15,16}$ In some cases where different lesion morphologies are present, a second or even multiple biopsies may aid in diagnosis; a more discrete and intact bulla was chosen as a second biopsy site. For other diagnostically challenging cases, KIT mutation analysis is recommended. ${ }^{12}$ Ultimately, the lack of growth in the blood culture, taken together with the Giemsa-positive mast cell proliferation in the second biopsy and negative DIF results, led us to the diagnosis of bullous mastocytosis.

\section{Management}

As there are no medications that alter disease course, the management of cutaneous mastocytosis revolves around prevention and symptomatic treatment. Avoiding triggers is an essential prevention method, giving caregivers one of the more crucial roles to play. Triggers include friction, dryness, physical exertion, sudden temperature changes, and certain medications (non-steroidal anti-inflammatory drugs, morphine). ${ }^{6}$ Infections, surgical/dental procedures, and vaccinations are likewise known to be triggers that may require premedication with antihistamines. ${ }^{2}$ Hence, the caregiver's awareness of these factors may directly impact the patient's quality of life.

Oral $\mathrm{H} 1, \mathrm{H} 2$ antihistamines, or mast cell stabilizers such as oral cromolyn sodium are first-line therapies for DCM. The addition of topical corticosteroids, topical calcineurin inhibitors, and anti-leukotrienes may be helpful if first-line medications are insufficient; oral corticosteroids may be given for more severe cases or those involving angioedema, abdominal pain, or diarrhea. ${ }^{6}$ Commonly, a combination of these medications is used to address the different symptoms of the disease; some authors used topical steroids in the morning, topical tacrolimus at night, and an antibiotic cream for eroded lesions. ${ }^{17}$ Others achieved resolution of bullae in a few days by combining oral $\mathrm{H} 1$ and $\mathrm{H} 2$ antihistamines and oral prednisolone. ${ }^{18}$ In our case, topical corticosteroid, oral $\mathrm{H} 1$ antihistamine (cetirizine), and bland emollients were sufficient to manage milder symptoms. However, oral prednisolone was occasionally required for relief during severe flares. Additionally, epinephrine autoinjectors should be available for all DCM patients as they have an increased risk for anaphylaxis. ${ }^{6}$

Prognosis is reportedly favorable for childhood cutaneous mastocytosis, with $50 \%$ of cases regressing; this contrasts with adult-onset disease, which may persist and become systemic.,19 While complications such as anaphylaxis and systemic involvement are rarely expected in pediatric cases. Physicians must still consider these possibilities and advise regular consultations to prevent severe episodes.

\section{CONCLUSION}

Diagnosis of diffuse cutaneous mastocytosis may be met with challenges due to its rarity. This case stresses the importance of having a high clinical suspicion, augmented by histopathologic findings, in arriving at a diagnosis. Once diagnosed, bullous mastocytosis requires prevention and symptomatic treatment, as well as communication of prognosis with the stakeholders.

\section{Acknowledgment}

Many thanks to the residents from co-managing services, Dr. Patricia Asuncion (Department of Pediatrics) and Dr. Picoy Cruz (Department of Surgery).

\section{Statement of Authorship}

All authors have substantial contributions to acquisition, analysis, interpretation of data; drafting the work; approval of the final version; agreement to be accountable to all aspects of the works.

\section{Author Disclosure}

All authors declared no conflicts of interest.

\section{Funding Support}

None.

\section{REFERENCES}

1. Janssens AS, Heide R, den Hollander JC, Mulder PGM, Tank $\mathrm{B}$, Oranje AP. Mast cell distribution in normal adult skin. J Clin Pathol. 2005 Mar;58(3):285-9.

2. Castells M, Metcalfe DD, Escribano L. Diagnosis and Treatment of Cutaneous Mastocytosis in Children. Am J Clin Dermatol. 2011 Aug 1;12(4):259-70.

3. Tharp M. Mastocytosis. In: Fitzpatrick's Dermatology in General Medicine. 8th ed. McGraw Hill Professional; 2012. p. 1809-18.

4. Méni C, Bruneau J, Georgin-Lavialle S, Le Saché de Peufeilhoux L, Damaj G, Hadj-Rabia S, et al. Paediatric mastocytosis: a systematic review of 1747 cases. Br J Dermatol. 2015 Mar;172(3):642-51.

5. Hannaford R, Rogers M. Presentation of cutaneous mastocytosis in 173 children. Australas J Dermatol. 2001 Feb;42(1):15-21.

6. Hosking A-M, Makdisi J, Ortenzio F, de Feraudy S, Smith J, Linden K. Diffuse cutaneous mastocytosis: Case report and literature review. Pediatr Dermatol. 2018 Nov;35(6):e348-52.

7. Philippine Dermatological Society - Health Information System. Cutaneous Mastocytosis. 2019.

8. Horny H, Metcalfe DD, Bennett J, Bain J, Akin C, Escribano L, et al. Mastocytosis. In: WHO Classification of Tumours of Haematopoietic and Lymphoid Tissues [Internet]. 4th ed. Lyon, France: International Agency for Research on Cancer; 2008 [cited 2019 Oct 25]. p. 54-63. Available from: https://publications.iarc.fr/Book-And-Report-Series/ Who-Iarc-Classification-Of-Tumours/Who-Classification-OfTumours-Of-Haematopoietic-And-Lymphoid-Tissues-2017

9. Valent P, Akin C, Metcalfe DD. Mastocytosis: 2016 updated WHO classification and novel emerging treatment concepts. Blood. 2017 Mar 16;129(11):1420-7.

10. Deverriere G, Square D, Nae I, Cailliez D, Boulloche J. Diffuse bullous mastocytosis of the infant: a rare clinical form - ScienceDirect [Internet]. 2012 [cited 2019 Feb 25]. Available from: https://www.sciencedirect. com/science/article/pii/S0929693X12002126?via\%3Dihub 
11. Srinivas S, Dhar S, Parikh D. Mastocytosis in children. Indian J Paediatr Dermatol. 2015;16(2):57-63.

12. Lange M, Lugowska-Umer H, Niedoszytko M, Wasag B, Limon J, Zawrocki A, et al. Diagnosis of Mastocytosis in Children and Adults in Daily Clinical Practice. Acta Dermatovenerol Croat [Internet]. 2015 Aug 11 [cited 2019 Mar 1];96(3). Available from: http://www. medicaljournals.se/acta/content/html/10.2340/00015555-2210

13. Goodlad J, Calonje E. Cutaneous lymphoproliferative diseases and related disorders. In: McKee's Pathology of the Skin with Clinical Correlations. 4th ed. China: Elsevier; 2012. p. 1413-20.

14. Tharp MD. Mastocytosis. In: Dermatology. 3rd ed. China: Elsevier Limited; 2012. p. 1993-2002.

15. Nischal U, Nischal KC, Khopkar U. Techniques of Skin Biopsy and Practical Considerations. J Cutan Aesthet Surg. 2008;1(2):107-11.
16. Cafardi J. The Manual of Dermatology. Springer Science \& Business Media; 2012. 585 p.

17. Avshalumov K, Pichardo R, Jorizzo J, Sangueza O, Goldenberg G. Bullous mastocytosis: report of a patient and a brief review of the literature. 2008 Oct [cited 2019 Feb 25]; Available from: https:// www.ncbi.nlm.nih.gov/pubmed/18806488

18. Ghosh A, Choudhury J, Dhar S. An infant with bullous mastocytosis: A rare form of bullous disorder. Indian J Paediatr Dermatol. 2017;18(2):122-4.

19. Chatterjee A, Ghosh J, Kapur R. Mastocytosis: a mutated KIT receptor-induced myeloproliferative disorder. Oncotarget. 2015 Jun 5;6(21):18250-64.

Have you read the current trends in Medical and Health Research in the Philippines?

\title{
Acta Medica Philippina The National Health Science Journal
}

\author{
Access Online: www.actamedicaphilippina.upm.edu.ph
}

